\title{
Elevated levels of inflammatory plasma biomarkers are associated with risk of HIV infection
}

\author{
Samantha Mclnally ${ }^{1}$, Kristin Wall ${ }^{2}$, Tianwei Yu ${ }^{3}$, Rabindra Tirouvanziam ${ }^{4,5}$, William Kilembe ${ }^{6}$, Jill Gilmour ${ }^{7}$,
} Susan A. Allen ${ }^{8}$ and Eric Hunter ${ }^{1,8^{*}}$ (1)

\begin{abstract}
Background: To determine if individuals, from HIV-1 serodiscordant couple cohorts from Rwanda and Zambia, who become HIV-positive have a distinct inflammatory biomarker profile compared to individuals who remain HIV-negative, we compared levels of biomarkers in plasma of HIV-negative individuals who either seroconverted (pre-infection) and became HIV-positive or remained HIV-negative (uninfected).

Results: We observed that individuals in the combined cohort, as well as those in the individual country cohorts, who later became HIV-1 infected had significantly higher baseline levels of multiple inflammatory cytokines/ chemokines compared to individuals who remained HIV-negative. Genital inflammation/ulceration or schistosome infections were not associated with this elevated profile. Defined levels of ITAC and IL-7 were significant predictors of later HIV acquisition in ROC predictive analyses, whereas the classical Th1 and Th2 inflammatory cytokines such as IL-12 and interferon- $\gamma$ or IL-4, IL-5 and II-13 were not.
\end{abstract}

Conclusions: Overall, the data show a significant association between increased plasma biomarkers linked to inflammation and immune activation and HIV acquisition and suggests that pre-existing conditions that increase systemic biomarkers represent a factor for increased risk of HIV infection.

Keywords: Cytokines, Chemokines, HIV pathogenesis, HIV acquisition, HIV discordant couples

\section{Background}

HIV-1 remains a major health crisis facing the world today. At the end of 2019, 37.9 million people were infected with HIV-1 globally [1]. It is well known that sex workers, men who have sex with men, intravenous drug users, and transgender individuals are at increased risk for HIV acquisition [1], as are the seronegative partners in HIV-1 discordant cohabiting couples [2]. Genital inflammation and ulceration clearly contributes to increased risk of HIV-1 acquisition. Studies in discordant

*Correspondence: ehunte4@emory.edu

${ }^{8}$ Department of Pathology and Laboratory Medicine, Emory University, Atlanta, GA, USA

Full list of author information is available at the end of the article couples in Rwanda and Zambia, as well as studies in other cohorts, have shown that individuals with genital ulceration/inflammation have a 5-10-fold higher risk for HIV infection [3-10]. Genital ulceration/inflammation has also been shown to affect the transmission bottleneck in heterosexual HIV-1 infection. The presence of genital inflammation has been shown to increase the likelihood of transmission of two or more transmittedfounder viruses, or multivariate transmission $[3,11]$. Sexually transmitted infections (STI) are frequently associated with increased genital inflammation. Schistosoma haematobium, which colonizes the venous plexus in the bladder, has also been shown to induce a similar increase in the risk of HIV acquisition [12-17]. In a study of the prevalence of Schistosomiasis antibodies in sera from a 
heterosexual HIV-discordant couple cohort in Lusaka, Zambia, it was found that Schistosome infections were linked to increased HIV-1 transmission in both sexes, increased acquisition of HIV-1 in women, and increased progression to death in HIV-positive women [18].

In a large study of at-risk HIV-negative women in South Africa it was observed that higher levels of inflammatory cytokines in the female genital tract of individuals prior to infection was associated with increased HIV susceptibility [6], allowing a more defined approach to quantitating this risk factor for acquisition. It is clear that in women the composition of the genital microbiome can also greatly influence the genital tract inflammatory state. Consistent with this notion, a study of the FRESH cohort in Durban, South Africa, showed that women who presented with Lactobacillus-deficient microbiota in their reproductive tract produced higher levels of inflammatory cytokines [19]. A follow-up study showed that these women had a greater risk of HIV acquisition compared to women with Lactobacillus crispatus-rich genital microbiota [20]. However, the genital cytokine levels observed were not correlated with those in the plasma [20].

In this study, we examined systemic plasma biomarker levels in individuals who would eventually seroconvert (pre-infection) and those who remained HIV-negative (uninfected) in a cohort of serodiscordant couples from both Zambia and Rwanda. We compared these two groups to determine if there was any association between systemic plasma biomarker levels and increased acquisition of HIV in HIV-negative individuals, and whether those biomarkers were associated with any pre-existing urogenital infections.

\section{Results}

Elevated systemic plasma cytokine and chemokine levels characterize individuals prior to infection

A major goal of this study was to determine whether prior to infection seroconverting partners in the two discordant couple cohorts under study exhibited a different inflammatory cytokine or chemokine profile compared to those who remained seronegative. Plasma from a total of 38 Zambian participants (19 uninfected, 19 preinfection) and 30 Rwandan participants (17 uninfected, 13 pre-infection) were analyzed in a Luminex multiplex assay. All of the individuals included in this study were negative partners in an HIV-1 serodiscordant couple, and were analyzed a median of $>1000$ days following enrolment (Zambia) and $>450$ days (Rwanda) (see Additional file 1: Table S1). Initial analyzes showed that the levels of the biomarkers were similar in the two countries, allowing an initial analysis of the combined Zambia and Rwanda cohorts (see Additional file 2: Figure S1). We observed that 18 of the 21 biomarkers measured were significantly increased in the pre-infection group compared to the uninfected group (Fractalkine, GMCSF, ITAC, IL-1ß, IL-2, IL-5, IL-6, IL-7, IL-8, IL-10, IL-12, IL17a, IL-21, IL-23, MIP-1 $\alpha$, MIP-1ß, MIP-3 $\alpha$, and TNF $\alpha$ ) (Fig. 1 and Additional file 3: Table S2). All p-values have undergone FDR-correction to account for the multiplicity of the assay. A majority of these upregulated cytokines and chemokines are involved in the inflammatory response. Because many of these cytokines are upregulated in coordinated pathways, we analyzed the data using a random forest model to establish those exerting most influence on the phenotype. Five biomarkers (ITAC, IL-8, IL-7, TNF $\alpha$, and Fractalkine) were identified as the most significant contributors to the signature associated with future HIV-1 infection (Fig. 2a). We also analyzed the data using a Partial Least Squares (PLS) analysis. The PLS showed that the levels of GMCSF, Fractalkine, IFNg, ITAC, IL-1ß, IL-2, IL-5, IL-7, IL-8, IL-10, IL-12, IL-17 $\alpha$, IL-21, IL-23, MIP-1ß, and TNF $\alpha$ had higher impact on the separation between the uninfected and the pre-infection cohort (Fig. 2b and Additional file 4: Table S3 A). This result confirms our initial analysis that multiple inflammatory biomarkers are significantly elevated in individuals who later become infected. To further interrogate these findings, we analyzed the data from the Zambia and Rwanda combined cohorts to try and determine if genital inflammation contributed to the elevated profile observed in the pre-infection cohort.

\section{Presence of genital inflammation or ulceration in the combined cohort does not explain elevated biomarker levels in pre-infection group}

Past studies of the Zambian and Rwandan cohorts, as well as other studies, have shown that the presence of genital inflammation/ulceration increases an individual's susceptibility to infection by HIV $[3,4,6-9,19,20]$. To test the possible role of genital inflammation or ulceration in the elevated biomarker profile, we compared the levels in individuals from the pre-infection group for whom any form of genital inflammation or ulceration had been reported in the 6 months prior to sample collection with those for whom no genital inflammation or ulceration was reported. There was no significant difference in the levels of any of the 21 analytes analyzed. Similarly, genital inflammation/ulceration did not significantly impact systemic biomarkers in the uninfected group. Moreover, after removing individuals with genital inflammation from the analysis, 11 of the biomarkers remained significantly higher in the pre-infection group compared to the uninfected group (Fractalkine, GMCSF, ITAC, IL-1ß, IL-6, IL-7, IL-8, IL-21, MIP- $1 \alpha$, MIP-3 $\alpha$, and TNF $\alpha$ ) (Fig. 3). We next analyzed the Rwandan and Zambian cohorts separately to determine whether any 


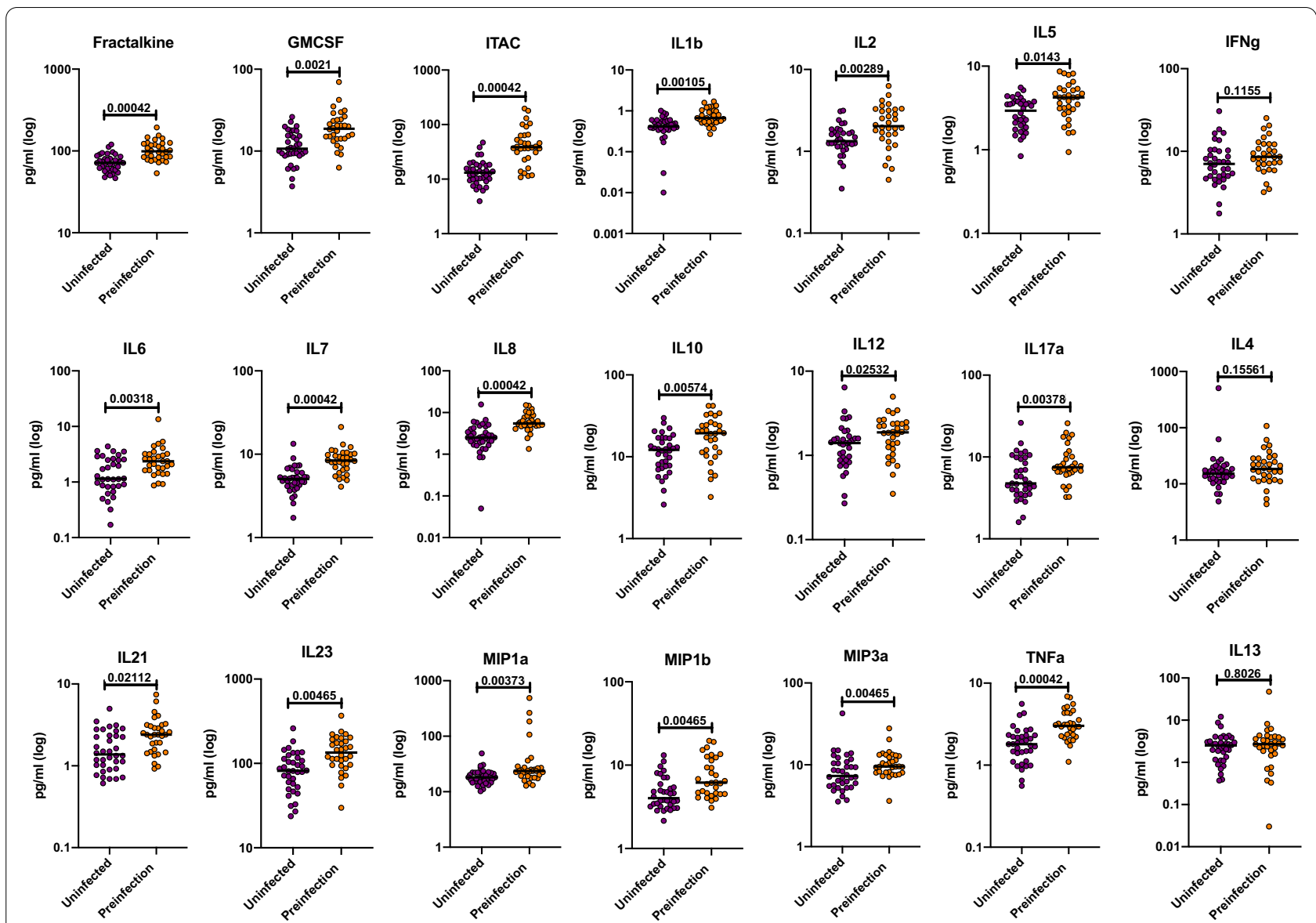

Fig. 1 Circulating biomarkers in the combined Rwanda and Zambia cohorts: preinfection individuals have significantly higher cytokines and chemokine levels compared to the uninfected group. Cytokine and chemokines concentrations from plasma from the preinfection and uninfected groups were compared (Kolmogorov-Smirnov test, two-tailed, FDR adjusted p-values). The uninfected (purple) and preinfection (orange) groups contained a mixture of samples from Zambia and Rwanda

differences existed between the two cohorts when analyzed in a similar manner.

\section{Elevated systemic plasma cytokine and chemokine levels associated with pre-infection individuals in both Zambian and Rwandan cohorts}

Despite the smaller numbers analyzed when the data was separated by country, we still observed that several inflammatory biomarkers were significantly higher in the pre-infection group than the uninfected group. In Rwanda, Fractalkine, GMCSF, ITAC, IL-1ß, IL-6, IL-7, IL-8, MIP- $1 \alpha$, and TNF $\alpha$ concentrations were significantly increased in the pre-infection group compared to the uninfected group (Fig. 4a and Additional file 5: Table S4A). The median, interquartile range, and $\mathrm{p}$-values for this analysis is shown in Additional file 5: Table S4A. When analyzed in a PLS model, Fractalkine, GMCSF, IFNg, ITAC, IL-1ß, IL-2, IL-5, IL-6, IL-7, IL-8, IL-10, IL-17 $\alpha$, IL-21, MIP-1 $\alpha$, MIP-1ß, and TNF $\alpha$ were found to be the major contributors to the separation of the groups (Fig. 4b and Additional file 4: Table S3B). This supports our initial univariate analysis of the Rwanda cohort since there were a number of additional biomarkers that were trending towards significance (see Additional file 6: Figure S2).

In Zambia, Fractalkine, ITAC, IL-7, IL-8, IL-23, and TNF $\alpha$ concentrations were significantly increased in the pre-infection group compared to the uninfected group in univariate comparisons (Fig. 5a and Additional file 7: Figure S3). The median, interquartile range, and p-values for this analysis are shown in Additional file 5: Table S4B. While elevated levels of Fractalkine, ITAC, IL-7, IL-8, and TNF $\alpha$ are observed for both countries in their respective pre-infection groups, they differ in that they exhibit additional biomarkers unique to each country. The PLS analysis showed that levels of ITAC, IL-5, IL-7, IL-8, and MIP-1 $\alpha$ were associated with pre-infection (Fig. $5 \mathrm{~b}$ and Additional file 4: Table S3C). 

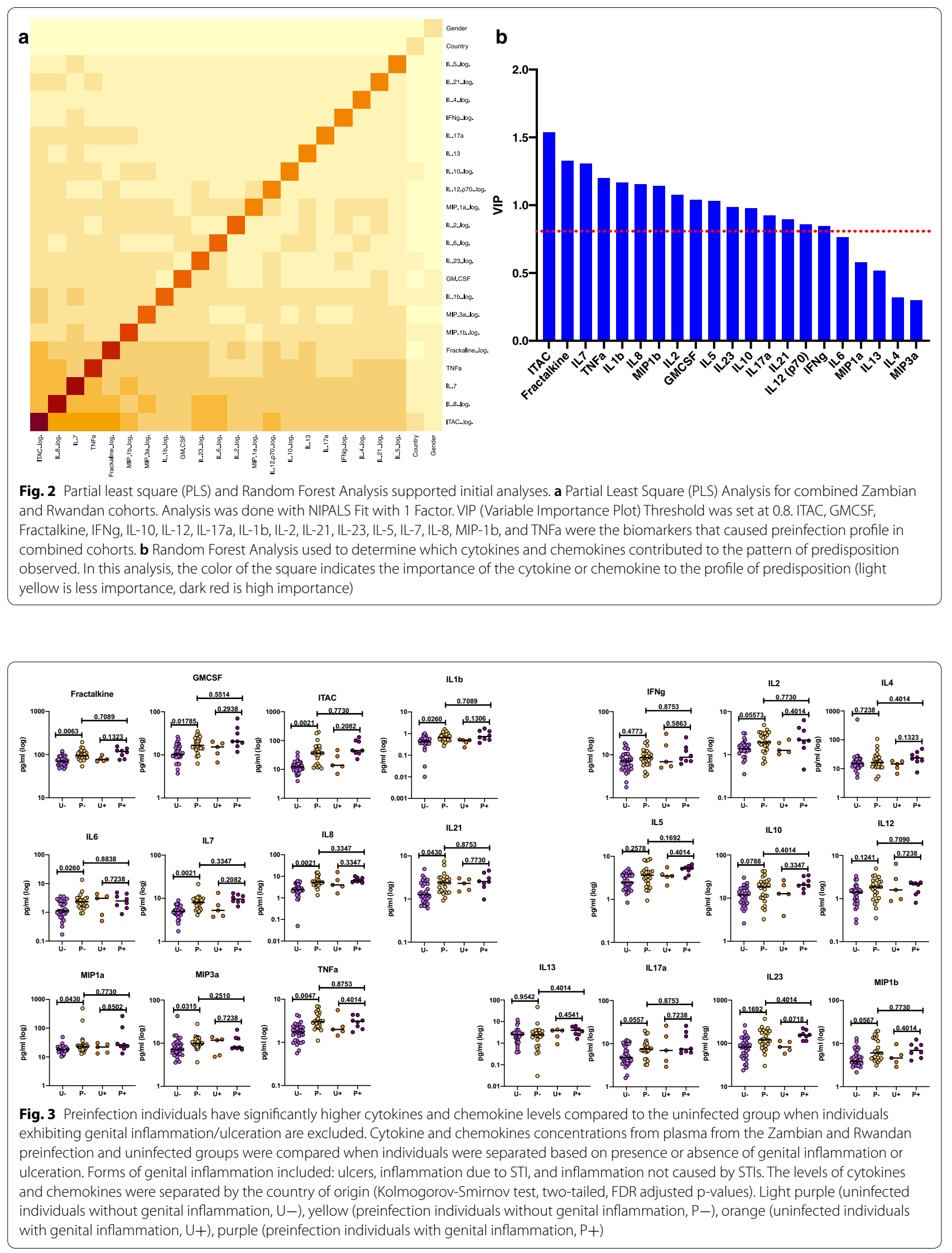


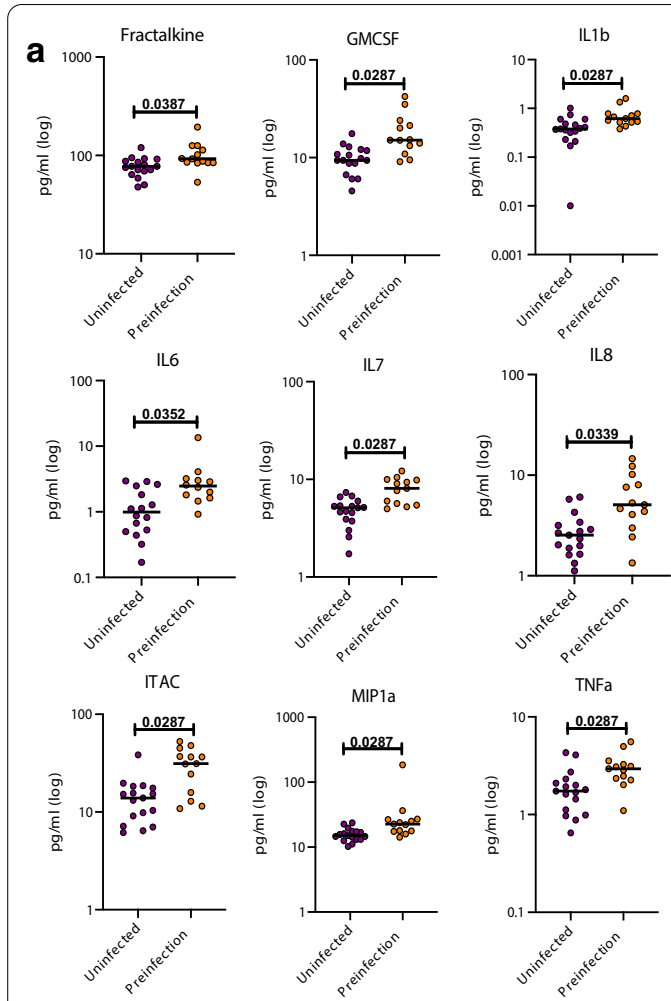

b

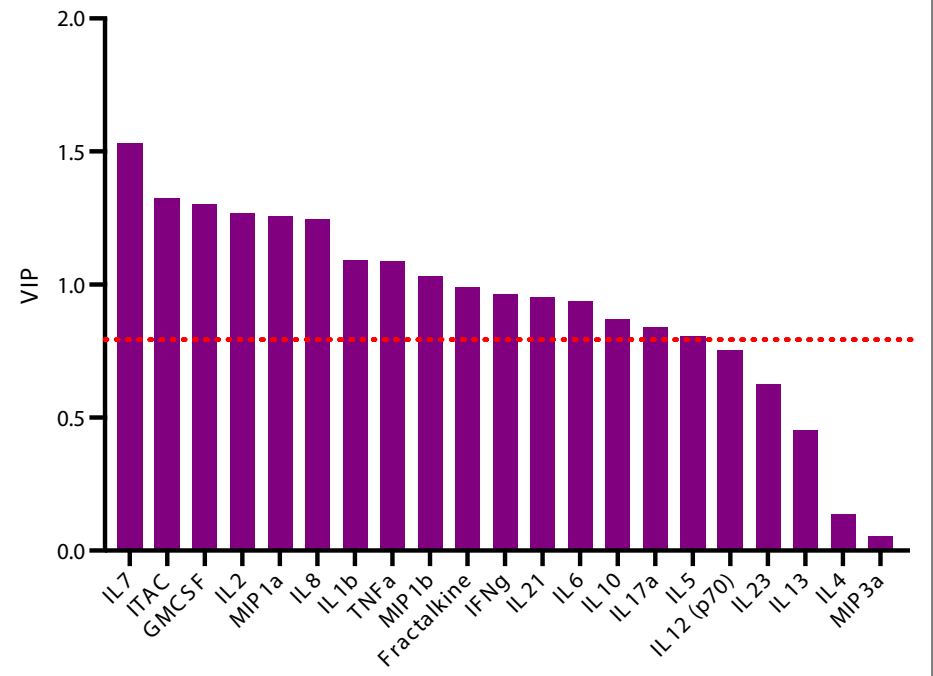

Fig. 4 Circulating biomarkers in the Rwanda cohort: preinfection individuals have significantly higher cytokines and chemokine levels compared to the uninfected group. a The levels of cytokines and chemokines were separated by the country of origin and the uninfected (purple) and preinfection (orange) groups were compared (Kolmogorov-Smirnov test, two-tailed, FDR adjusted p-values). b Partial Least Square (PLS) Analysis for the Rwandan cohort. Analysis was done with NIPALS Fit with 1 Factor. VIP (Variable Importance Plot) Threshold was set at 0.8. PLS analysis for Rwandan cohort. ITAC, GMCSF, Fractalkine, IFNg, IL-10, IL-17a, IL-1 b, IL-2, IL-21, IL-5, IL-6, IL-7, IL-8, MIP-1a, MIP-1b, and TNFa were the biomarkers that caused preinfection profile in Rwanda

Taken together, we observed that the pre-infection group had elevated biomarker levels in both Rwanda and Zambia even when the cohorts are analyzed separately.

\section{Presence of Schistosomiasis antibody titers does not appear to be a major contributor to the elevated biomarker profile in pre-infected individuals}

We have recently reported that infection with Schistosoma haematobium is common even in urban settings in Zambia and that infection was associated with increased susceptibility to HIV-1 infection [18]. Because this parasite colonizes the venous plexus in the bladder, we investigated prior infection as evidenced by antibodies resulted in elevated cytokines. However, screening of the plasma from both the uninfected and pre-infection groups for antibodies to the schistosome showed that a similar proportion of individuals in both had detectable antibody titers (9/19 uninfected; 11/19 pre-infection). The Zambian cohort was therefore divided into four groups: uninfected individuals with negative Schistosomiasis antibody titers, uninfected individuals with positive antibody titers, pre-infection individuals with negative titers, and pre-infection individuals with positive titers (see Additional file 8: Figure S4). We did not observe any significant differences in biomarker levels between seropositive and seronegative individuals in either the uninfected group or the pre-infection group. With the proviso that we are analyzing very small groups of individuals, this suggests that schistosome infection is not associated with the elevated biomarker levels observed in the preinfection group.

\section{Predictive analyses identify elevated levels of several biomarkers as markers of pre-infection}

Based on preliminary results from partial least squares analysis (Figs. 2b, 4b and 5b), we wanted to determine which biomarkers included in the study were associated with future HIV-1 acquisition. To achieve this, we analyzed the combined Zambian and Rwandan cohort using Receiver Operating Characteristics (ROC) curves and established a cutoff for the area under the curve (AUC) of 0.8 . Using a cutoff of 0.8 means that any biomarker identified in the analysis has over an $80 \%$ chance of distinguishing pre-infection individuals from those that remain 

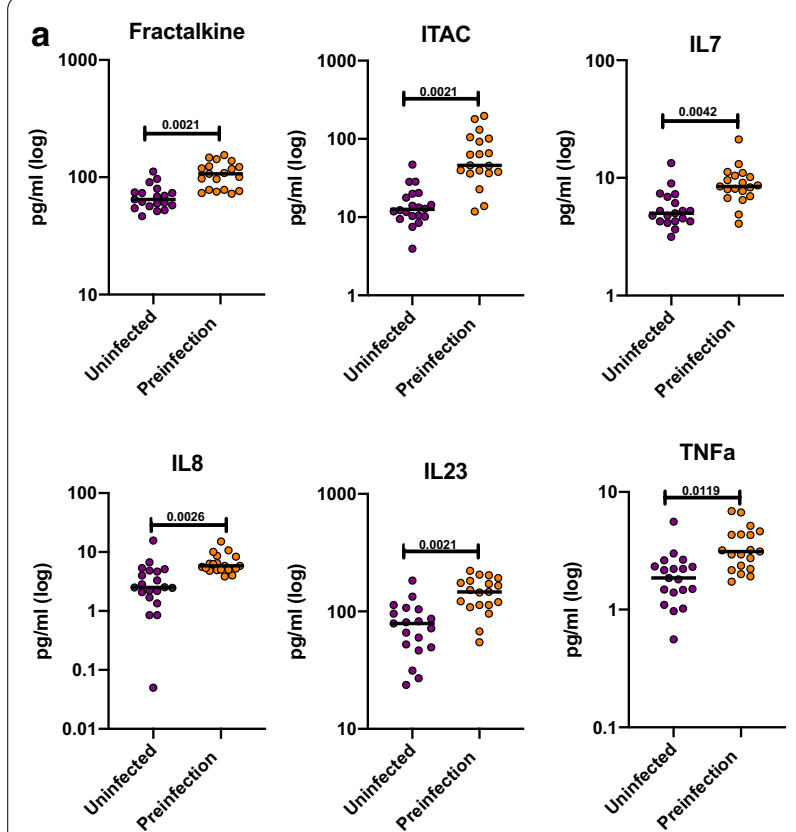

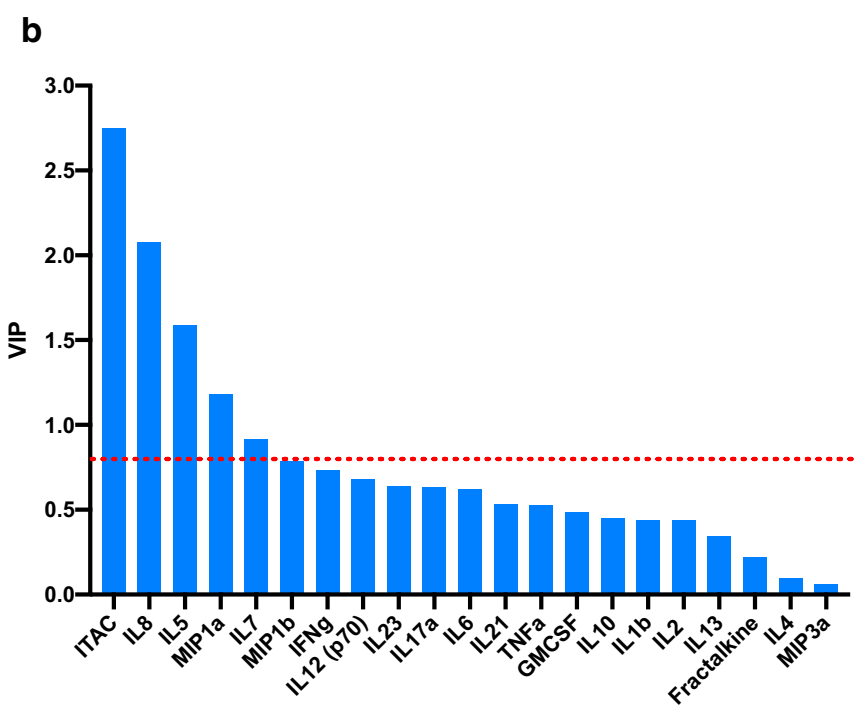

Fig. 5 Circulating biomarkers in the Zambia cohort: preinfection individuals have significantly higher cytokines and chemokine levels compared to the uninfected group. a The levels of cytokines and chemokines were separated by the country of origin and the uninfected (purple) and preinfection (orange) groups were compared (Kolmogorov-Smirnov test, two-tailed, FDR adjusted p-values). b Partial Least Square (PLS) Analysis for the Zambian cohort. Analysis was done with NIPALS Fit with 1 Factor. VIP (Variable Importance Plot) Threshold was set at 0.8. a PLS analysis for Zambian cohort. ITAC, IL-5, IL-8, and MIP-1a were the biomarkers that caused preinfection profile in Zambia

uninfected. ITAC, Fractalkine, IL-7, IL-8, and TNF $\alpha$ were identified as markers predictive for pre-infection (Fig. 6). ROC curves for individual Zambia and Rwanda cohorts are shown in Additional files 9 and 10: Figures S5 and S6. This finding is supported by our random forest analysis (Fig. 2a). Using a kfold validation model, we found that concentrations of ITAC above $30.98 \mathrm{pg} / \mathrm{ml}$ was highly predictive of a seronegative partner who would become infected (probability of 0.91 ; sensitivity of 0.75 ; specificity 0.94). If an IL-7 value above $6.99 \mathrm{pg} / \mathrm{ml}$ was added to the model, the probability of identifying pre-infection individuals increased to 0.97 (Fig. 7).

\section{Discussion}

This study found that individuals who will eventually seroconvert have higher levels of proinflammatory biomarkers in their plasma compared to individuals who remain HIV-negative, suggesting a novel link between predisposition to HIV infection and systemic biomarker levels.
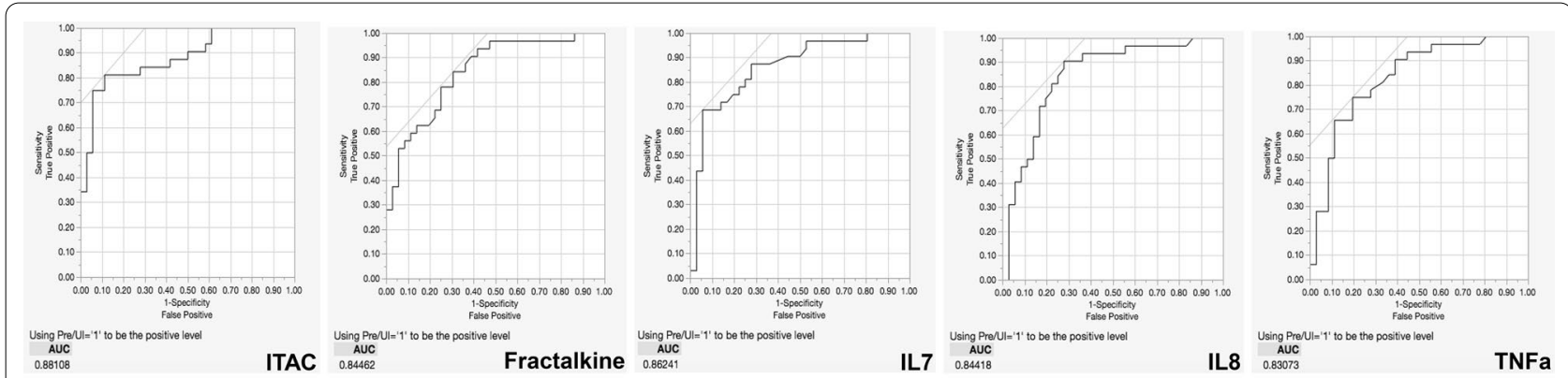

Fig. 6 Receiver Operating Characteristics (ROC) curves for combined Zambia and Rwanda cohorts identifies biomarkers that distinguishes preinfection individuals. Elevated levels of ITAC, Fractalkine, IL-7, IL-8, and TNFa identify individuals as risk for HIV acquisition. Area under the curve (AUC) shut off was 0.8 for separating the uninfected and preinfection individuals 


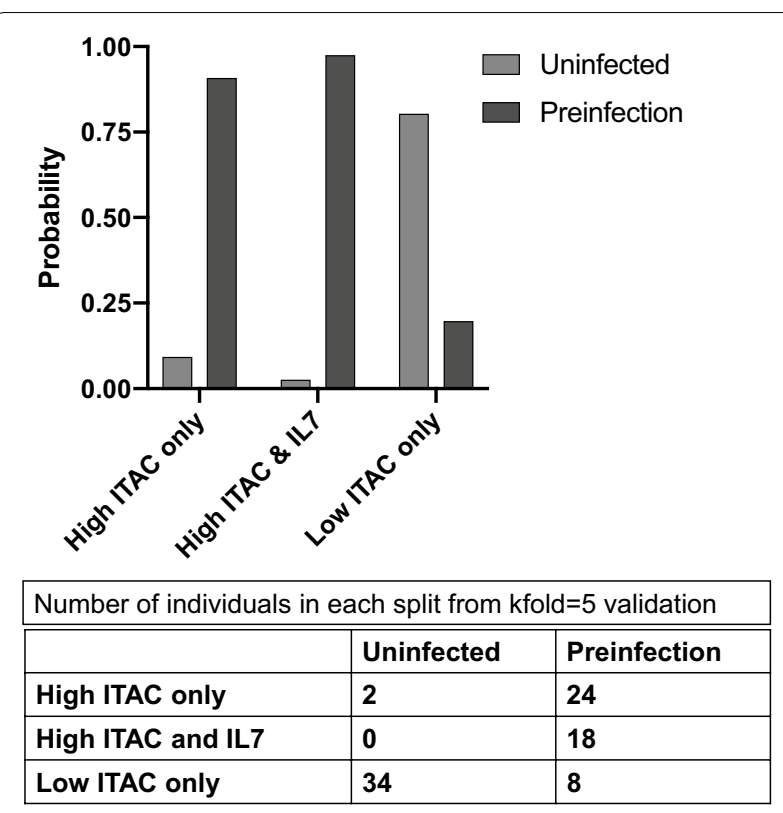

Fig. 7 Kfold validation model identifies elevated ITAC and IL-7 levels as markers for preinfection from combined Rwandan and Zambian cohorts. Two splits were performed to find biomarkers associated with preinfection. The bar graph shows the response probability for the multiple splits performed in the model. The model found that elevated ITAC identified preinfection individuals with a probability of 0.9077 and elevated ITAC and IL-7 identified preinfection individuals with a probability of 0.9744 . Lower levels of ITAC were found identify uninfected individuals. Uninfected (gray) and preinfection (maroon). The table shows the number of individuals from the total cohort that fall into each split performed by the model $(k f o l d=5)$

Our findings suggest that pre-existing conditions which induce systemic inflammation can represent a risk factor for HIV acquisition.

An example of a viral infection capable of inducing this is hepatitis $C$ virus. Plasma levels of IL-10 and MIP1ß were positively correlated with HCV RNA levels and may be involved in HCV immunopathogenesis [21], HIV/HCV co-infected women had higher levels of several proinflammatory biomarkers - biomarkers elevated in each infection or disease discussed in this section are summarized in Additional file 11: Table S5. In addition, HCV-positive HIV-negative women had higher levels of IFNg and IL-17 compared to other groups [22]. However, the prevalence of $\mathrm{HCV}$ infections in both Zambia and Rwanda has been reported to be low [23, 24].

Elevated systemic levels of cytokines and chemokines are also seen in multiple infections common in sub-Saharan Africa. Multiple researchers have found that malaria infection has been linked to elevated systemic cytokine responses. HIV and P. falciparum co-infected individuals had increased HIV viral loads, a steeper decline in $\mathrm{CD} 4^{+}$ $\mathrm{T}$ cell counts, and exhibited higher levels of several acute phase proinflammatory cytokines [25]. Similarly, symptomatic malaria infections had increased levels of multiple cytokines compared to uninfected controls [26]. It has also been found that individuals with greater malaria disease severity had higher levels of CRP, TNF $\alpha$, and IFNg [27]. P. vivax infections also are associated with higher levels of proinflammatory cytokines [28].

Elevated systemic biomarkers have also been found in Tuberculosis (TB). Multiple studies found that individuals with acute and latent TB infections had higher levels of cytokines and chemokines compared to healthy controls [29-32]. These results show that TB infections can increase several proinflammatory cytokines regardless of the stage of infection.

A separate study looked at proinflammatory cytokine levels in TB-infected individuals co-infected with S. stercoralis. S. stercoralis is a soil-transmitted helminth that infects about 50-100 million people worldwide [33]. Individuals co-infected with $\mathrm{TB}$ and $S$. stercoralis have increased levels of several type 2 cytokines compared to individuals only infected with TB [34]. One possible explanation for this is an association with microbial translocation. S. stecoralis infected individuals exhibited significantly higher plasma levels of microbial translocation markers (i.e. LPS) and this was associated with increased levels of several proinflammatory cytokines [35]. Researchers looking at microbial translocation in HIV infected individuals found that LPS was also positively correlated with plasma levels of IL-6, TNF $\alpha$, and hsCRP [36]. This finding is particularly interesting because it is well known that microbial translocation is associated with disease severity in HIV infections [37].

These studies show that several common infections in sub-Saharan Africa are capable of elevating systemic levels of several pro-inflammatory cytokines, which we found as a risk factor for HIV acquisition. However, it is also possible that higher levels of biomarkers prior to infection may have additional impact once an individual becomes HIV-infected. High levels of several cytokines appear to enhance HIV replication and disease progression [38]. Additional studies are needed not only to determine the cause of the elevated levels of biomarkers in individuals prior to HIV acquisition, but also how these elevated levels may impact the disease progression of these patients once infected.

While these common infections in Africa are associated with elevated inflammatory cytokines, the observed biomarker profile of the pre-infection individuals reported on here is not indicative of a classic innate immune response or $\mathrm{T}$ cell response. Indeed, the elevated cytokines and chemokines observed play roles in many different arms of the immune system. Our analysis showed that three biomarkers, Fractalkine, ITAC, and 
IL-7, were highly predictive as risk factors for HIV acquisition if elevated above certain levels. These biomarkers can be elevated from infection, but also from non-communicable conditions. Compared to healthy controls, elevated levels of Fractalkine were found in individuals with type 2 diabetes, systemic lupus erythematosus, and systemic sclerosis, an autoimmune disorder that affects the vascular system and leads to early defective angiogenesis. [39-42]. In systemic sclerosis, the higher levels of soluble Fractalkine in the blood were associated with vascular activation and increased disease severity [42]. That same study found higher levels of ITAC compared to healthy controls [42]. ITAC, also known as IFN-inducible $\mathrm{T}$ cell $\alpha$ chemoattractant, was found to be at higher levels in patients with inflammatory bowel disease (Crohn's disease and ulcerative colitis), fibromyalgia, and sarcoidosis [43-45]. Sarcoidosis is a systemic inflammatory granulomatous disease that affects lungs of its patients and higher systemic levels of ITAC were found to be predictive of future pulmonary function test decline [43].

IL-7 is not normally considered an inflammatory cytokine and is more associated with $\mathrm{T}$ cell homeostasis. However, higher levels of IL-7 were found to be associated with several diseases, such was fibromyalgia [46] and also in patients with colorectal and esophageal cancers compared to healthy controls [47]. Unfortunately, health data on such conditions were not recorded as part of the Heterosexual Transmission Protocol into which discordant couples were enrolled. Nevertheless, the studies reported here indicate that additional studies of cytokine/chemokine levels in at risk individuals is warranted.

There are a number of limitations to the current study. We were only able to analyze the inflammatory biomarkers in a limited number of individuals since the availability of pre-HIV infection plasma samples from the discordant couples studied here are also limited (only $3-7 \%$ of individuals in this cohort seroconverted per year depending on the cohort). In addition, we have limited information on other infections and health conditions of individuals in study. This is compounded by the fact that only a small number of studies have reported on systemic biomarkers in other infections or diseases in the context of an African population. As a result, it is difficult for us to identify specific infections or diseases that may be causing the elevated biomarker profile that we observed. Analogous studies of cohorts in the USA or Europe, where additional biomarker data on other infections/diseases may be available, would represent a valuable follow-up study to this one.

The identification of key biomarkers associated with HIV acquisition has important clinical ramifications. Our study identified elevated levels of both ITAC and IL-7 as highly predictive of HIV acquisition risk. If individuals in a HIV high-risk region are found to have elevated levels of one or both of these biomarkers, more intensive HIV-1 prevention approaches could be taken in order to protect those individuals. Additional studies are needed not only to determine the cause of the elevated levels of biomarkers in individuals prior to HIV acquisition and how they facilitate the acquisition event, but also how these elevated levels may impact the disease progression of these patients once infected. Overall, our findings suggest that individuals at risk for infection could be identified by testing for elevated levels of a very limited number of biomarkers.

\section{Conclusions}

We show that in a cohort of seronegative partners from serodiscordant couples, individuals who eventually acquire HIV from their partner have higher levels of inflammatory cytokines and chemokines compared to individuals that remain HIV-negative. This was observed in both Rwanda and Zambia where subtype A and subtype $\mathrm{C}$ are the predominant HIV-1 serotypes. A Receiver Operator Characteristics analysis showed that the levels of just two cytokines, ITAC and IL-7, were highly predictive of future infection. This suggests high systemic biomarker levels are both a risk factor and a quantitative predictor for HIV acquisition.

\section{Materials and methods \\ Study Subjects}

All participants were enrolled in the Rwanda Zambia HIV Research Group (RZHRG) discordant couple cohorts in Lusaka, Zambia and Kigali, Rwanda. Subjects from both cohorts were enrolled in human subjects protocols approved by the Emory Institutional Review Board, the Rwanda National Ethics Committee and the University of Zambia Research Ethic Committee and provided written consent. When the participants enrolled in the cohort, they were provided couples counseling and testing, treatment for sexually transmitted infections (STIs), and condoms to reduce transmission of HIV-1. All the subjects tested in this study were the seronegative partner within a serodiscordant cohabitating heterosexual couple. Subjects were selected on their seronegative status, availability of plasma samples, and selected from both Zambian and Rwandan cohorts. The negative partner was tested for HIV every 1 to 3 months. In the Zambia cohort, the median days from enrollment to when the sample was taken was 1234 for the uninfected group and 1087 for the pre-infection group. Samples for the pre-infection group was collected a median of 46 days before the estimated date of infection (EDI). In the Rwanda cohort, the median days from enrollment to when the samples were 
taken was 494 for the uninfected group and 457 for the pre-infection group. Samples for the pre-infection group was collected a median of 45 days before the EDI. The algorithm used to determine the EDI has been previously described [3].

\section{Evaluation of plasma cytokines}

The plasma cytokine and chemokine levels were measured using a Milliplex Map Human High Sensitivity T Cell Panel (HSTCMAG-28SK). This kit measures the levels of 21 inflammatory cytokines and chemokines. The samples were run in duplicate. In order to eliminate batch to batch variation in the assay, all tests were carried out on the same batch of plates and approximately equal numbers of pre-infection and uninfected plasma were run on the same plate. The plates were quantified and standardized on a Bioplex 2000 at the Yerkes Virology Core and final concentrations were extrapolated from a standard curve and expressed in $\mathrm{pg} / \mathrm{ml}$. All plasma samples were stored at $-80{ }^{\circ} \mathrm{C}$ and had undergone a single freeze-thaw for aliquoting prior to use.

\section{Genital inflammation and ulceration data collection}

As described in Haaland et al. [3], medical and laboratory signs and symptoms of inflammatory or ulcerative STI, candida, and bacterial vaginosis were recorded systematically at routine study visits and at interim sick visits, with full physical and/or genital exams conducted annually and as clinically indicated; physical and genital exams were routinely conducted on the visit date when lab test results indicated HIV-1 seroconversion. A self-reported symptom was considered present whether or not the patient sought medical treatment and included treatment administered at external clinics. The generation of the composite variables were described in Wall et al. [4]. Briefly, for each 3-monthly interval, composite variables were created. The genital inflammation composite included inflammatory STIs (clinical or laboratory diagnosis or treatment of gonorrhea, chlamydia or trichomonas) and non-inflammatory STIs (reported discharge, dysuria, dyspareunia; observed discharge or inflammation of external or internal genitalia; and/or laboratory diagnosis of candida or BV). The composite for genital ulcer included observed or reported genital ulcers and/ or incident positive RPR. A subject was considered having positive genital inflammation or ulceration if they had presence of either in the 6 months prior to biomarker sample collection.

\section{Schistosomiasis antibody titer data collection}

As described in Wall et al. [18], plasma samples were collected at baseline analyzed in an ELISA assay for antibodies to schistosome soluble worm antigen preparation (SWAP). A 4-parameter curve fitting model was used to assign units based on the standard curve to each unknown plasma. The positive cutoff value was set at three standard deviations above the average anti-SWAP IgG in serum from egg negative controls from the US and Europe. A positive schistosomiasis result was defined as having a positive SWAP antibody response.

\section{Data analysis}

Comparison between the uninfected and the pre-infection groups were done with nonparametric Kolmogorov-Smirnov tests in Prism 9. We addressed the multiple testing issue by using the Benjamini-Hochberg False Discovery Rate (FDR) correction [48]. The Random Forest model was performed in $\mathrm{R} 4.0 .0$ at the default setting. The details were described previously [49].

Partial Least Squares (PLS) analysis was performed using the JMP Pro 15 statistical package. PLS analysis had a variable importance cutoff of 0.8 . The Receiver Operating Characteristic (ROC) curve analysis used a cutoff of 0.8 for area under the curve (AUC). The predictive results were generated with a $\mathrm{k}$-fold validation which included two splits in the model $(\mathrm{k}=5)$.

\section{Supplementary Information}

The online version contains supplementary material available at https://doi. org/10.1186/s12977-021-00552-6.

\section{Acknowledgements \\ We acknowledge all participating volunteers in Zambia and Rwanda as well as the researchers at the Rwanda Zambia HIV Research Group who made this project possible. We thank Charlott Morel Sanchez, Lelah Harmon, and Reese Tierney for sample management, Paul Farmer for sample repository and database management, W. Evan Secor for parasite data assistance, and David Lee for Luminex assistance.}

\section{Authors' contributions}

Conceived and designed the experiments: SM, RT, EH. Performed the experiments: SM. Provided genital inflammation/ulceration and parasite data: KW. Analyzed the data: SM, TY. Contributed reagents/materials/analysis: SM, KW, TY, RT, JG, WK, SA, EH. Wrote the paper: SM, EH. All authors read and approved the final manuscript.

\section{Funding}

This study was funded by Grants R01 Al 51231 (to E.H.), R01 Al (to E.H.), and F31 Al 145750 (to S.M.) from the National Institute of Allergy and Infectious Diseases, National Institutes of Health. This work was also supported, in part, by the Virology Core at the Emory Center for AIDS Research by performing the Luminex assays (grant P30 Al050409) and the Yerkes National Primate Research Center base grant through the Office of Research Infrastructure Programs/OD P51OD11132. It was also supported in part by the International AIDS Vaccine Initiative (S.A.), whose work is make possible by support from many donors, including the Bill \& Melinda Gates Foundation, the Ministry of Foreign Affairs of Denmark, Irish Aid, the Ministry of Finance of Japan, the Ministry of Foreign Affairs of Netherlands, the Norwegian Agency for Development Cooperation, the UK Department for International Development, and the US Agency for International Development (USAID). The full list of IAVI donors is available. E.H. is a Georgia Eminent Scholar. 


\section{Availability of data and materials}

Source data available on request.

\section{Declarations}

\section{Ethics approval and consent to participate}

This study was approved by the Emory Institutional Review Board, the Rwanda National Ethics Committee and the University of Zambia Research Ethic Committee.All participants gave written informed consent for their enrollment in the cohort.

\section{Consent for publication}

Not applicable.

\section{Competing interests}

The authors declare no competing interests.

\begin{abstract}
Author details
1 Emory Vaccine Center at Yerkes National Primate Research Center, Atlanta, GA, USA. ${ }^{2}$ Rollins School of Public Health, Emory University, Atlanta, GA, USA. ${ }^{3}$ School of Data Science, The Chinese University of Hong Kong, Shenzhen, Shenzhen, Guangdong Province, China. ${ }^{4}$ Department of Pediatrics, Emory University School of Medicine, Atlanta, GA, USA. ${ }^{5}$ Center of CF and Airways Disease Research, Children's Healthcare of Atlanta, Atlanta, GA, USA. ${ }^{6}$ ZambiaEmory HIV Research Project, Lusaka, Zambia. ${ }^{7}$ Faculty of Medicine, Imperial College, London SW7 2AZ, UK. ${ }^{8}$ Department of Pathology and Laboratory Medicine, Emory University, Atlanta, GA, USA.
\end{abstract}

Received: 21 December 2020 Accepted: 6 March 2021 Published online: 17 March 2021

\section{References}

1. UNAIDS Data. 2019 [Internet]2019 [cited 2020].

2. Wall KM, Kilembe W, Vwalika B, Haddad LB, Lakhi S, Onwubiko U, Htee Khu N, Brill I, Chavuma R, Vwalika C, Mwananyanda L, Chomba E, Mulenga J, Tichacek A, Allen S. Sustained effect of couples' HIV counselling and testing on risk reduction among Zambian HIV serodiscordant couples. Sex Transm Infect. 2017;93(4):259-66. https://doi.org/10.1136/ sextrans-2016-052743 Epub 2017/01/14.

3. Haaland RE, Hawkins PA, Salazar-Gonzalez J, Johnson A, Tichacek A, Karita E, Manigart O, Mulenga J, Keele BF, Shaw GM, Hahn BH, Allen SA, Derdeyn CA, Hunter E. Inflammatory genital infections mitigate a severe genetic bottleneck in heterosexual transmission of subtype A and C HIV-1. PLoS Pathog. 2009;5(1):e1000274. https://doi.org/10.1371/journal.ppat.10002 74.

4. Wall KM, Kilembe W, Vwalika B, Haddad LB, Hunter E, Lakhi S, Chavuma R, Htee Khu N, Brill I, Vwalika C, Mwananyanda L, Chomba E, Mulenga J, Tichacek A, Allen S. Risk of heterosexual HIV transmission attributable to sexually transmitted infections and non-specific genital inflammation in Zambian discordant couples, 1994-2012. Int J Epidemiol. 2017;46(5):1593-606. https://doi.org/10.1093/ije/dyx045.

5. Hope TJ. Inflammation weakens HIV prevention. Nat Med. 2018;24(4):3845. https://doi.org/10.1038/nm.4534.

6. Masson L, Passmore JA, Liebenberg LJ, Werner L, Baxter C, Arnold KB, Williamson C, Little F, Mansoor LE, Naranbhai V, Lauffenburger DA, Ronacher K, Walzl G, Garrett NJ, Williams BL, Couto-Rodriguez M, Hornig M, Lipkin WI, Grobler A, Abdool Karim Q, Abdool Karim SS. Genital inflammation and the risk of HIV acquisition in women. Clin Infect Dis. 2015;61(2):260-9. https://doi.org/10.1093/cid/civ298.

7. Passmore JS, Jaspan HB. Vaginal microbes, inflammation, and HIV risk in African women. Lancet Infect Dis. 2018;18(5):483-4. https://doi.org/10. 1016/S1473-3099(18)30061-6.

8. Roberts L, Passmore JA, Mlisana K, Williamson C, Little F, Bebell LM, Walzl G, Abrahams MR, Woodman Z, Abdool Karim Q, Abdool Karim SS. Genital tract inflammation during early HIV-1 infection predicts higher plasma viral load set point in women. J Infect Dis. 2012;205(2):194-203. https:// doi.org/10.1093/infdis/jir715.
9. Sabo MC, Lehman DA, Wang B, Richardson BA, Srinivasan S, Osborn L, Matemo D, Kinuthia J, Fiedler TL, Munch MM, Drake AL, Fredricks DN, Overbaugh J, John-Stewart G, McClelland RS, Graham SM. Associations between vaginal bacteria implicated in HIV acquisition risk and proinflammatory cytokines and chemokines. Sex Transm Infect. 2020;96(1):3-9. https://doi.org/10.1136/sextrans-2018-053949.

10. Malhotra R, Hu L, Song W, Brill I, Mulenga J, Allen S, Hunter E, Shrestha S, Tang J, Kaslow RA. Association of chemokine receptor gene (CCR2-CCR5) haplotypes with acquisition and control of HIV-1 infection in Zambians. Retrovirology. 2011;8:22. https://doi.org/10.1186/1742-4690-8-22.

11. Carlson JM, Schaefer M, Monaco DC, Batorsky R, Claiborne DT, Prince J, Deymier MJ, Ende ZS, Klatt NR, DeZiel CE, Lin TH, Peng J, Seese AM, Shapiro R, Frater J, Ndung'u T, Tang J, Goepfert P, Gilmour J, Price MA, Kilembe W, Heckerman D, Goulder PJ, Allen TM, Allen S, Hunter E. HIV transmission. Selection bias at the heterosexual HIV-1 transmission bottleneck. Science. 2014;345(6193):1254031. https://doi.org/10.1126/science.1254031.

12. Mbabazi PS, Andan O, Fitzgerald DW, Chitsulo L, Engels D, Downs JA. Examining the relationship between urogenital schistosomiasis and HIV infection. PLoS Negl Trop Dis. 2011;5(12):e1396. https://doi.org/10.1371/ journal.pntd.0001396.

13. Downs JA, Dupnik KM, van Dam GJ, Urassa M, Lutonja P, Kornelis D, de Dood CJ, Hoekstra P, Kanjala C, Isingo R, Peck RN, Lee MH, Corstjens P, Todd J, Changalucha JM, Johnson WD Jr, Fitzgerald DW. Effects of schistosomiasis on susceptibility to HIV-1 infection and HIV-1 viral load at HIV-1 seroconversion: A nested case-control study. PLoS Negl Trop Dis. 2017;11(9):e0005968. https://doi.org/10.1371/journal.pntd.0005968.

14. Downs JA, van Dam GJ, Changalucha JM, Corstjens PL, Peck RN, de Dood CJ, Bang H, Andreasen A, Kalluvya SE, van Lieshout L, Johnson WD Jr, Fitzgerald DW. Association of Schistosomiasis and HIV infection in Tanzania. Am J Trop Med Hyg. 2012;87(5):868-73. https://doi.org/10.4269/ ajtmh.2012.12-0395.

15. Kjetland EF, Ndhlovu PD, Mduluza T, Gomo E, Gwanzura L, Mason PR, Kurewa EN, Midzi N, Friis H, Gundersen SG. Simple clinical manifestations of genital Schistosoma haematobium infection in rural Zimbabwean women. Am J Trop Med Hyg. 2005;72(3):311-9.

16. Leutscher PD, Pedersen M, Raharisolo C, Jensen JS, Hoffmann S, Lisse I, Ostrowski SR, Reimert CM, Mauclere P, Ullum H. Increased prevalence of leukocytes and elevated cytokine levels in semen from Schistosoma haematobium-infected individuals. J Infect Dis. 2005;191(10):1639-47. https://doi.org/10.1086/429334.

17. Sawers L, Stillwaggon E, Hertz T. Cofactor infections and HIV epidemics in developing countries: implications for treatment. AIDS Care. 2008;20(4):488-94. https://doi.org/10.1080/09540120701868311.

18. Wall KM, Kilembe W, Vwalika B, Dinh C, Livingston P, Lee YM, Lakhi S, Boeras D, Naw HK, Brill I, Chomba E, Sharkey T, Parker R, Shutes E, Tichacek A, Secor WE, Allen S. Schistosomiasis is associated with incident HIV transmission and death in Zambia. PLoS Negl Trop Dis. 2018;12(12):e0006902. https://doi.org/10.1371/journal.pntd.0006902.

19. Anahtar MN, Byrne EH, Doherty KE, Bowman BA, Yamamoto HS, SoumilIon M, Padavattan N, Ismail N, Moodley A, Sabatini ME, Ghebremichael MS, Nusbaum C, Huttenhower C, Virgin HW, Ndung'u T, Dong KL, Walker $B D$, Fichorova RN, Kwon DS. Cervicovaginal bacteria are a major modulator of host inflammatory responses in the female genital tract. Immunity. 2015;42(5):965-76.

20. Gosmann C, Anahtar MN, Handley SA, Farcasanu M, Abu-Ali G, Bowman BA, Padavattan N, Desai C, Droit L, Moodley A, Dong M, Chen Y, Ismail N, Ndung'U T, Ghebremichael MS, Wesemann DR, Mitchell C, Dong KL, Huttenhower C, Walker BD, Virgin HW, Kwon DS. Lactobacillus-deficient cervicovaginal bacterial communities are associated with increased HIV acquisition in Young South African Women. Immunity. 2017:46(1):29-37. https://doi.org/10.1016/j.immuni.2016.12.013.

21. Hajarizadeh B, Lamoury FM, Feld JJ, Amin J, Keoshkerian E, Matthews GV, Hellard M, Dore GJ, Lloyd AR, Grebely J, Applegate TL, Group AS. Alanine aminotransferase, HCV RNA levels and pro-inflammatory and pro-fibrogenic cytokines/chemokines during acute hepatitis $C$ virus infection. Virol J. 2016;13:32. https://doi.org/10.1186/s12985-016-0482-x

22. Fernandez-Botran $R$, Joshi-Barve $S$, Ghare $S$, Barve S, Young M, Plankey $\mathrm{M}$, Bordon J. Systemic cytokine and interferon responsiveness Patterns in HIV and HCV mono and co-infections. J Interferon Cytokine Res. 2014;34(11):885-93 10.1089/jir.2014.0006. 
23. Mohd Hanafiah K, Groeger J, Flaxman AD, Wiersma ST. Global epidemiology of hepatitis $C$ virus infection: new estimates of age-specific antibody to HCV seroprevalence. Hepatology. 2013;57(4):1333-42. https://doi.org/ 10.1002/hep.26141.

24. Mora N, Adams WH, Kliethermes S, Dugas L, Balasubramanian N, Sandhu J, Nde H, Small C, Jose J, Scaglione S, Layden JE. A Synthesis of Hepatitis C prevalence estimates in Sub-Saharan Africa: 2000-2013. BMC Infect Dis. 2016;16:283. https://doi.org/10.1186/s12879-016-1584-1.

25. Goncalves RM, Lima NF, Ferreira MU. Parasite virulence, co-infections and cytokine balance in malaria. Pathog Glob Health. 2014;108(4):173-8. https://doi.org/10.1179/2047773214Y.0000000139.

26. Goncalves RM, Scopel KK, Bastos MS, Ferreira MU. Cytokine balance in human malaria: does Plasmodium vivax elicit more inflammatory responses than Plasmodium falciparum? PLoS ONE. 2012;7(9):e44394. https://doi.org/10.1371/journal.pone.0044394.

27. Andrade BB, Reis-Filho A, Souza-Neto SM, Clarencio J, Camargo LM, Barral A, Barral-Netto M. Severe Plasmodium vivax malaria exhibits marked inflammatory imbalance. Malar J. 2010;9:13. https://doi.org/10.1186/ 1475-2875-9-13.

28. Hojo-Souza NS, Pereira DB, de Souza FS, de Oliveira Mendes TA, Cardoso MS, Tada MS, Zanini GM, Bartholomeu DC, Fujiwara RT, Bueno LL. On the cytokine/chemokine network during Plasmodium vivax malaria: new insights to understand the disease. Malar J. 2017;16(1):42. https://doi.org/ 10.1186/s12936-017-1683-5.

29. Luo J, Zhang M, Yan B, Li F, Guan S, Chang K, Jiang W, Xu H, Yuan T, Chen $M$, Deng S. Diagnostic performance of plasma cytokine biosignature combination and MCP-1 as individual biomarkers for differentiating stages Mycobacterium tuberculosis infection. J Infect. 2019;78(4):281-91. https://doi.org/10.1016/j.jinf.2018.10.017.

30. Wu J, Wang S, Lu C, Shao L, Gao Y, Zhou Z, Huang H, Zhang Y, Zhang W. Multiple cytokine responses in discriminating between active tuberculosis and latent tuberculosis infection. Tuberculosis (Edinb). 2017;102:68-75. https://doi.org/10.1016/j.tube.2016.06.001.

31. Won EJ, Choi JH, Cho YN, Jin HM, Kee HJ, Park YW, Kwon YS, Kee SJ. Biomarkers for discrimination between latent tuberculosis infection and active tuberculosis disease. J Infect. 2017;74(3):281-93. https://doi.org/10. 1016/j.jinf.2016.11.010.

32. Clifford V, Tebruegge M, Zufferey C, Germano S, Forbes B, Cosentino L, Matchett E, McBryde E, Eisen D, Robins-Browne R, Street A, Denholm J, Curtis N. Cytokine biomarkers for the diagnosis of tuberculosis infection and disease in adults in a low prevalence setting. Tuberculosis (Edinb). 2019;114:91-102. https://doi.org/10.1016/j.tube.2018.08.011.

33. Anuradha R, Munisankar S, Bhootra Y, Jagannathan J, Dolla C, Kumaran P, Shen K, Nutman TB, Babu S. Systemic Cytokine Profiles in Strongyloides stercoralis Infection and Alterations following Treatment. Infect Immun. 2016;84(2):425-31. https://doi.org/10.1128/IAl.01354-15.

34. Kathamuthu GR, Munisankar S, Sridhar R, Baskaran D, Babu S. Helminth mediated modulation of the systemic and mycobacterial antigen - stimulated cytokine profiles in extra-pulmonary tuberculosis. PLoS Negl Trop Dis. 2019;13(3):e0007265. https://doi.org/10.1371/journal.pntd.0007265.

35. Rajamanickam A, Munisankar S, Bhootra Y, Dolla C, Nutman TB, Babu S. Microbial translocation associated with an acute-phase response and elevations in MMP-1, HO-1, and proinflammatory cytokines in Strongyloides stercoralis Infection. Infect Immun. 2017. https://doi.org/10.1128/IAI. 00772-16.

36. Dinh DM, Volpe GE, Duffalo C, Bhalchandra S, Tai AK, Kane AV, Wanke CA, Ward HD. Intestinal microbiota, microbial translocation, and systemic inflammation in chronic HIV infection. J Infect Dis. 2015;211(1):19-27. https://doi.org/10.1093/infdis/jiu409.
37. Marchetti G, Tincati C, Silvestri G. Microbial translocation in the pathogenesis of HIV infection and AIDS. Clin Microbiol Rev. 2013;26(1):2-18. https:// doi.org/10.1128/CMR.00050-12.

38. Chatterjee A, Rathore A, Vidyant S, Kakkar K, Dhole TN. Chemokines and chemokine receptors in susceptibility to HIV-1 infection and progression to AIDS. Dis Markers. 2012;32(3):143-51. https://doi.org/10.3233/ DMA-2011-0874.

39. Benyamine A, Magalon J, Cointe S, Lacroix R, Arnaud L, Bardin N, Rossi P, Frances Y, Bernard-Guervilly F, Kaplanski G, Harle JR, Weiller PJ, Berbis P, Braunstein D, Jouve E, Lesavre N, Couranjou F, Dignat-George F, Sabatier F, Paul P, Granel B. Increased serum levels of Fractalkine and mobilisation of CD34(+)CD45(-) endothelial progenitor cells in systemic sclerosis. Arthritis Res Ther. 2017;19(1):60. https://doi.org/10.1186/s13075-017-1271-7.

40. Guo L, Lu X, Wang Y, Bao C, Chen S. Elevated levels of soluble Fractalkine and increased expression of CX3CR1 in neuropsychiatric systemic lupus erythematosus. Exp Ther Med. 2017;14(4):3153-8. https://doi.org/10. 3892/etm.2017.4862.

41. Sindhu S, Akhter N, Arefanian H, Al-Roub AA, Ali S, Wilson A, Al-Hubail A, Al-Beloushi S, Al-Zanki S, Ahmad R. Increased circulatory levels of Fractalkine (CX3CL1) are associated with inflammatory chemokines and cytokines in individuals with type-2 diabetes. J Diabetes Metab Disord. 2017;16:15. https://doi.org/10.1186/s40200-017-0297-3.

42. Crescioli C, Corinaldesi C, Riccieri V, Raparelli V, Vasile M, Del Galdo F, Valesini G, Lenzi A, Basili S, Antinozzi C. Association of circulating CXCL10 and CXCL11 with systemic sclerosis. Ann Rheum Dis. 2018;77(12):1845-6. https://doi.org/10.1136/annrheumdis-2018-213257.

43. Arger NK, Ho M, Woodruff PG, Koth LL. Serum CXCL11 correlates with pulmonary outcomes and disease burden in sarcoidosis. Respir Med. 2019;152:89-96. https://doi.org/10.1016/j.rmed.2019.04.005.

44. Garcia JJ, Cidoncha A, Bote ME, Hinchado MD, Ortega E. Altered profile of chemokines in fibromyalgia patients. Ann Clin Biochem. 2014;51(Pt 5):576-81. https://doi.org/10.1177/0004563213506413.

45. Singh UP, Singh NP, Murphy EA, Price RL, Fayad R, Nagarkatti M, Nagarkatti PS. Chemokine and cytokine levels in inflammatory bowel disease patients. Cytokine. 2016;77:44-9. https://doi.org/10.1016/j.cyto.2015.10. 0082015.10 .008$.

46. Backryd E, Tanum L, Lind AL, Larsson A, Gordh T. Evidence of both systemic inflammation and neuroinflammation in fibromyalgia patients, as assessed by a multiplex protein panel applied to the cerebrospinal fluid and to plasma. J Pain Res. 2017;10:515-25. https://doi.org/10.2147/JPR. S128508.

47. Bednarz-Misa I, Diakowska D, Krzystek-Korpacka M. Local and systemic IL-7 concentration in gastrointestinal-tract cancers. Medicina. 2019. https://doi.org/10.3390/medicina55060262.

48. Benjamini Y, Hochberg Y. Controlling the false discovery rate - a practical and powerful approach to multiple testing. J Roy Stat Soc B Met. 1995;57(1):289-300.

49. Monaco DC, Dilernia DA, Fiore-Gartland A, Yu T, Prince JL, Dennis KK, Qin K, Schaefer M, Claiborne DT, Kilembe W, Tang J, Price MA, Farmer P, Gilmour J, Bansal A, Allen S, Goepfert P, Hunter E. Balance between transmitted HLA preadapted and nonassociated polymorphisms is a major determinant of HIV-1 disease progression. J Exp Med. 2016;213(10):204963. https://doi.org/10.1084/jem.20151984.

\section{Publisher's note}

Springer Nature remains neutral with regard to jurisdictional claims in published maps and institutional affiliations. 